\title{
Challenging the concept of adding more drugs in pulmonary arterial hypertension
}

\author{
Caio J. C. dos Santos Fernandes (1) ${ }^{1}$, Marc Humbert (10) ${ }^{2,3,4}$ and Rogerio Souza (1) \\ Affiliations: ${ }^{1}$ Pulmonary Circulation Unit, Heart Institute, University of São Paulo Medical School, São \\ Paulo, Brazil. ${ }^{2}$ University Paris-Sud, Université Paris-Saclay, Le Kremlin Bicêtre, Paris, France. ${ }^{3}$ Service de \\ Pneumologie, Hôpital Bicêtre, Assistance Publique-Hôpitaux de Paris (AP-HP), Le Kremlin Bicêtre, Paris, \\ France. ${ }^{4}$ Inserm UMR-S 999, Hôpital Marie Lannelongue, Le Plessis Robinson, Paris, France.
}

Correspondence: R. Souza, Pulmonary Circulation Unit, Heart Institute, University of São Paulo Medical School, Av. Dr. Enéas de Carvalho Aguiar, 44-Pinheiros, São Paulo, 05403-900, Brazil.

E-mail: rogerio.souzađafm.usp.br

@ERSpublications

A "switch" strategy might represent an alternative for well-selected patients in PAH http://ow.ly/ppEt30enWo0

Cite this article as: dos Santos Fernandes CJ , Humbert M, Souza R. Challenging the concept of adding more drugs in pulmonary arterial hypertension. Eur Respir J 2017; 50: 1701527 [https://doi.org/10.1183/ 13993003.01527-2017].

\begin{abstract}
The management of pulmonary arterial hypertension (PAH) has evolved significantly in the last three decades based on the development of drugs which target three biochemical pathways involved in pulmonary vascular homeostasis: the nitric oxide (NO) pathway, the endothelin pathway and the prostacyclin pathway [1]. Initially employed as monotherapies, these new drugs changed PAH outcomes and had a direct impact on survival [2]. However, monotherapy proved over time to be insufficient for the vast majority of $\mathrm{PAH}$ patients and the next natural step in decreasing the still unacceptable lethality of $\mathrm{PAH}$ was the association of two or more drugs targeting distinct pathways. Results from two large randomised controlled trials demonstrated that the addition of a second or third drug in combination with an already established background therapy could significantly slow disease progression [3, 4], reinforcing the concept that more drugs can lead to greater benefit. This concept was further stressed by studying the upfront use of combination therapy. In the ambrisentan and tadalafil in patients with pulmonary arterial hypertension (AMBITION) trial [5], the combined use of an endothelin receptor antagonist (ERA) and a phosphodiesterase-5 inhibitor (PDE5i) proved to be better than the use of either compound alone in decreasing the risk of clinical failure events. Successful use of initial double or triple combination therapies was also reported in multi-centre registry studies $[6,7]$. These large trials and registries provided the basis for the current European Society of Cardiology (ESC)/European Respiratory Society (ERS) guidelines in which the use of combination therapy should be considered early, according to the stratification of the risk of death [8] (figure 1).
\end{abstract}

However, if a PAH patient fails to reach a meaningful response with a specific drug, is add-on always the right course of action? What if, instead of adding different drugs, the current drug is switched to another targeting the same pathway, or, perhaps, to a drug targeting a different pathway? How safe and effective would such an approach be? In this issue of the European Respiratory Journal, Hoeper et al. [9] report on the RESPITE study and switching to riociguat in PAH patients with inadequate response to a PDE5i. In this multi-centre single-arm study, $61 \mathrm{PAH}$ patients previously treated with a PDE5i $66 \%$ sildenafil and $34 \%$ tadalafil) who did not present a satisfactory response (as defined by persistent New York Heart

Received: July 262017 | Accepted: July 272017

Conflict of interest: Disclosures can be found alongside this article at erj.ersjournals.com

Copyright OERS 2017 

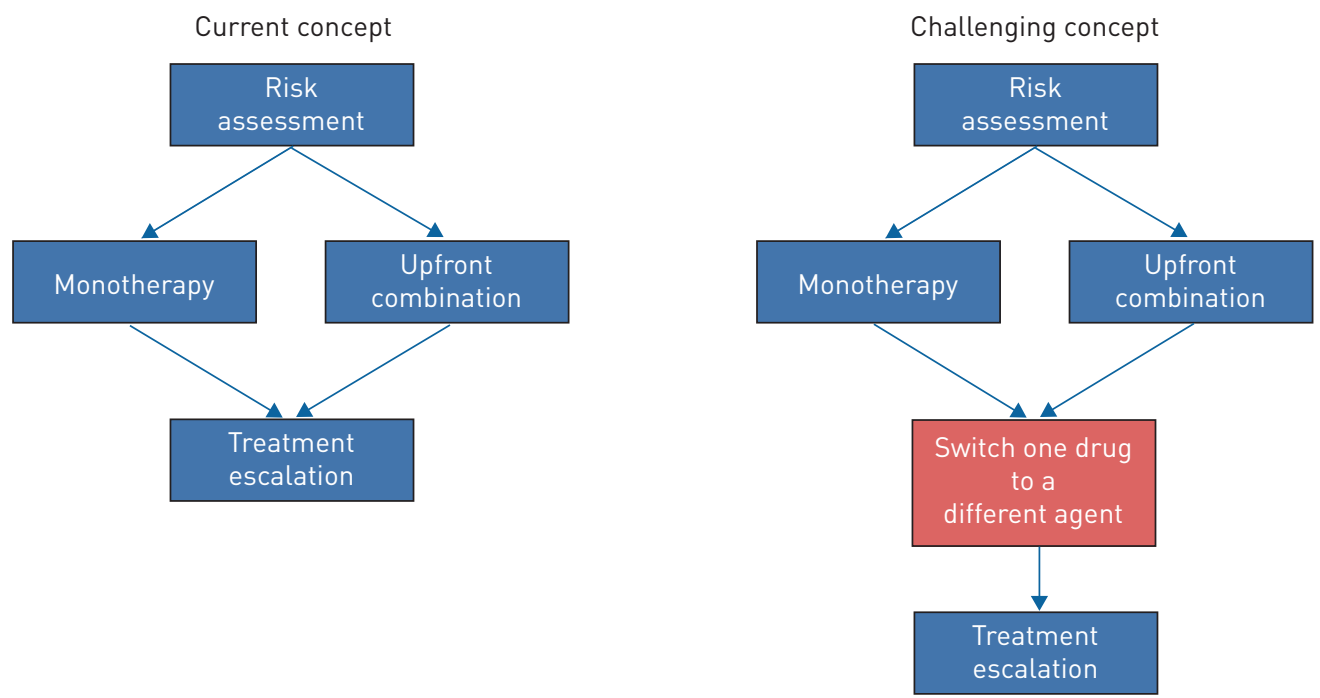

FIGURE 1 Different concepts for management of pulmonary arterial hypertension (PAH).

Association functional class III (FC III), a 6-min walking distance (6-MWD) of 165-440 m, a cardiac index of $<3.0 \mathrm{~L} \cdot \mathrm{min}^{-1} \cdot \mathrm{m}^{-2}$ and a pulmonary vascular resistance of $>400 \mathrm{dyn} \cdot \mathrm{s} \cdot \mathrm{cm}^{-5}$ ) were switched to riociguat, a soluble guanylate cyclase ( $\mathrm{sGC}$ ) stimulator which also targets the nitric oxide (NO) pathway but through a different molecular mechanism (sensitising the sGC to endogenous NO by enhancing NOsGC binding as opposed to directly stimulating the sGC via a different binding site independent of NO) $[6,8]$. At the end of the study, $84 \%$ of patients had completed the intended 24 weeks and, after riociguat therapy, had increased $6-\mathrm{MWD}$ by $31 \pm 63 \mathrm{~m} \quad(\mathrm{p}=0.0010)$, decreased $\mathrm{N}$-terminal pro-brain natriuretic peptide (NT-proBNP) by $347 \pm 1235 \mathrm{pg} \cdot \mathrm{mL}^{-1}(\mathrm{p}=0.0170)$ and significantly improved FC ( $54 \%$ of patients, $\mathrm{p}<0.0001)$. In the 49 patients with available haemodynamic re-evaluation data, pulmonary vascular resistance was reduced by $103 \pm 296 \mathrm{dyn} \cdot \mathrm{s} \cdot \mathrm{cm}^{-5}(\mathrm{p}=0.0184)$ and cardiac index increased by $0.3 \pm 0.5 \mathrm{~L} \cdot \mathrm{min}^{-1} \cdot \mathrm{m}^{-2}$ $(\mathrm{p}=0.0001)$. It is noteworthy that post hoc analysis showed that $51 \%$ of patients reached a lower risk profile (defined by the authors as the presence of more than $50 \%$ of available variables within the low-risk stratum of the risk stratification from the ESC/ERS guidelines [8]), while at baseline only $15 \%$ presented in this condition. On the other hand, $16 \%$ of patients did not complete the study, mostly due to side effects or clinical worsening. Notably, $16 \%$ of patients presented hypotension and ten experienced clinical worsening events, with two deaths during the 24 weeks. The effect of riociguat was independent of the concomitant use of an ERA ( $82 \%$ of patients) or previous type of PDE5i therapy.

The strategy of switching agents exists in many clinical settings: systemic hypertension being a clear example where, if significant control is not reached, the change to a different agent might be attempted before the addition of a second [10]. There are many difficulties that have limited the use of this strategy in PAH. First, there is no clear superiority between the different therapies that could support a stepwise treatment approach, even within the same pharmacological class. The exception to this is the use of an intravenous prostacyclin, which is widely considered to be the most efficacious but invasive approach to targeting the prostacyclin pathway. Secondly, there is no known profile for patients who respond better to one class of drugs than to another. In this sense, due to the chosen study design, RESPITE does not add to the identification of patients that might better benefit from the switch to riociguat from a PDE5i (a fact acknowledged by the authors). Lastly, PAH mortality remains high despite aggressive approaches with different therapy combinations and this prevents broader attempts at switching due to the risk of losing the optimal window for treatment in which a combination of different agents might be more effective.

Even considering these difficulties attempts have already been made at testing switch strategies in PAH management albeit in small cohorts or case series. Figure 2 summarises the possible combinations and potential switches tested in PAH. For example, Sitbon et al. [11] transitioned 12 patients from intravenous epoprostenol to intravenous treprostinil and all patients reported fewer prostacyclin-related side effects and remained on treprostinil after completion of the study; Coons et al. [12] transitioned nine stable PAH patients on parenteral or inhaled prostacyclins to oral treprostinil, with successful results in six cases; and Lichtblau et al. [13] performed a retrospective analysis of 13 patients who were switched from sildenafil to tadalafil due to side effects and described a successful result in $46.1 \%$ of patients. Furthermore, Safdar et al. [14] described a switch from bosentan to macitentan, in a retrospective study with 24 patients, which resulted in clinical stability. The same strategy was studied by Politi et al. [15] in a prospective cohort of 


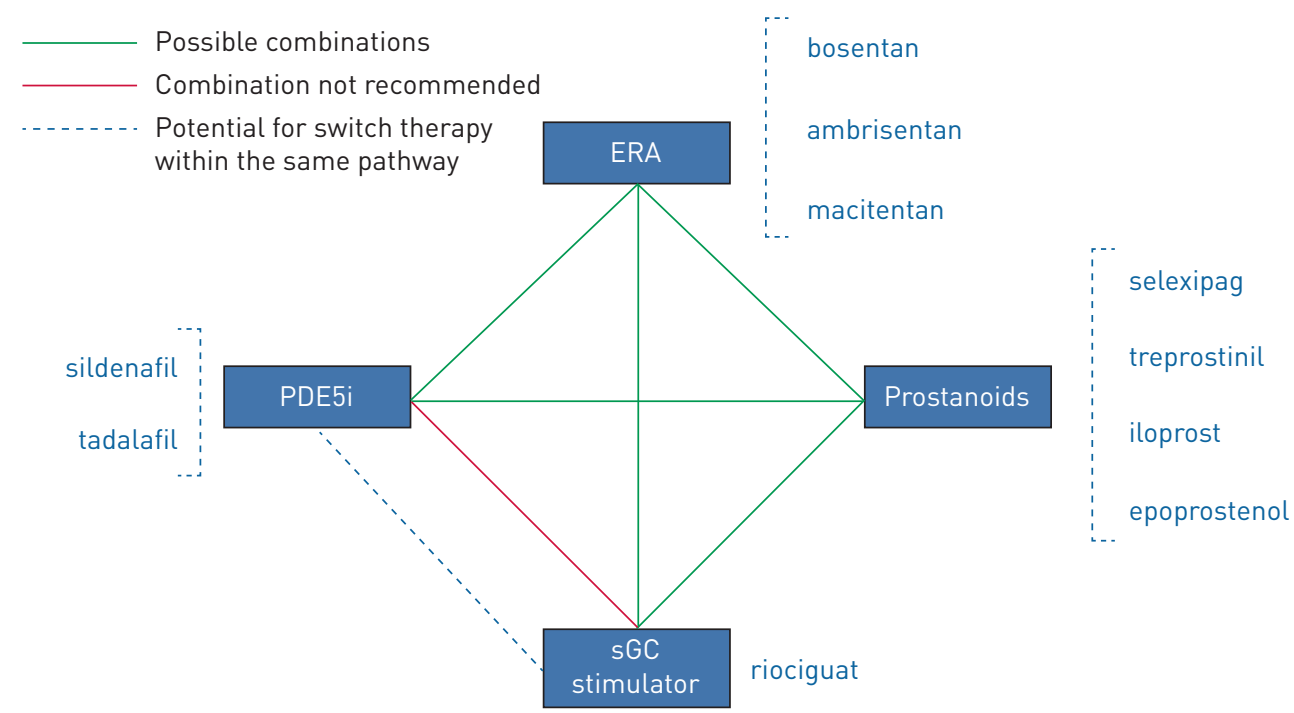

FIGURE 2 Pulmonary arterial hypertension (PAH) treatment combinations. ERA: endothelin receptor antagonist; PDE5i: phosphodiesterase-5 inhibitor; sGC: soluble guanylate cyclase.

21 patients transitioning from bosentan to macitentan, resulting in a significant improvement in FC, in 6-MWD and in NT-proBNP levels.

All these studies shared a common feature, namely that the switch was attempted with drugs from the same pharmacological class. What makes RESPITE unique in this setting is that the switched therapies, although aiming at the same pathway (in this case NO), constitute different classes of drugs that cannot themselves be combined due to the risk of severe hypotension, as already demonstrated [16]. However, RESPITE also has some important limitations that need to be acknowledged. First, the open-label design does not allow for a conclusion to be drawn, in the absence of a control group maintained with a PDE5i therapy, about the real role of switching from a PDE5i to riociguat. Secondly, a significant proportion of patients $(16 \%)$ did not complete the 24 weeks of the study, suggesting that the adverse event profile is relevant and may have influenced the final outcome. Indeed, when a sensitivity analysis is performed including the patients that did not complete the study, the only improvement that remains statistically significant is in FC. These sensitivity analyses do not invalidate the results of the study but do highlight the need for a better designed controlled trial before any suggestion of switching from a PDE5i therapy to riociguat is considered. Nevertheless, despite its limitations, RESPITE raises some relevant questions for the PAH field. Should we study other switches between drugs aimed at different pathways, or remain with the add-on/early combination strategy? Are there superior combinations that should be considered as first choices? Can we identify biomarkers or clinical profiles that identify better responses to one class of drugs or another?

RESPITE is a first-step, hypothesis-generating study which challenges current paradigms of PAH management. Its results should not be used as a basis for immediate modification of clinical PAH practice but rather to stimulate new randomised controlled studies to evaluate the different patient profiles that respond differently to a given therapy, providing the background for a more individualised treatment approach in PAH. The Riociguat rEplacing PDE5i therapy evaLuated Against Continued PDE5i thErapy (REPLACE) study (https://clinicaltrials.gov/ct2/show/NCT02891850), a prospective open-label, randomised, multi-centre, double-arm, controlled study of riociguat in patients with $\mathrm{PAH}$, who are on a stable dose of a PDE5i with or without an ERA but are not at treatment goal, is currently recruiting participants and will attempt to further evaluate the effectiveness of riociguat as a replacement for PDE5i therapy in PAH.

\section{References}

1 Humbert M, Sitbon O, Simonneau G. Treatment of pulmonary arterial hypertension. N Engl J Med 2004; 351: $1425-1436$

2 Humbert M, Simonneau G, Rubin LJ. A decade of achievement in pulmonary hypertension. Eur Respir Rev 2011; 20: 215-217.

3 Pulido T, Adzerikho I, Channick RN, et al. Macitentan and morbidity and mortality in pulmonary arterial hypertension. N Engl J Med 2013; 369: 809-818.

4 Sitbon O, Channick R, Chin KM, et al. Selexipag for the treatment of pulmonary arterial hypertension. $N$ Engl J Med 2015; 373: 2522-2533. 
5 Galie N, Barbera JA, Frost AE, et al. Initial use of ambrisentan plus tadalafil in pulmonary arterial hypertension N Engl J Med 2015; 373: 834-844.

6 Sitbon O, Jais X, Savale L, et al. Upfront triple combination therapy in pulmonary arterial hypertension: a pilot study. Eur Respir J 2014; 43: 1691-1697.

7 Sitbon O, Sattler C, Bertoletti L, et al. Initial dual oral combination therapy in pulmonary arterial hypertension. Eur Respir J 2016; 47: 1727-1736.

8 Galie N, Humbert M, Vachiery JL, et al. 2015 ESC/ERS Guidelines for the diagnosis and treatment of pulmonary hypertension. Eur Respir J 2015; 46: 903-975.

9 Hoeper MM, Simonneau G, Corris PA, et al. RESPITE: switching to riociguat in pulmonary arterial hypertension patients with inadequate response to phosphodiesterase-5 inhibitors. Eur Respir J 2017; 50: 1602425.

10 Mancia G, Fagard R, Narkiewicz K, et al. 2013 ESH/ESC Practice guidelines for the management of arterial hypertension. Eur Heart J 2013; 34: 2159-2219.

11 Sitbon O, Manes A, Jais X, et al. Rapid switch from intravenous epoprostenol to intravenous treprostinil in patients with pulmonary arterial hypertension. J Cardiovasc Pharmacol 2007; 49: 1-5.

12 Coons JC, Miller T, Simon MA, et al. Oral treprostinil for the treatment of pulmonary arterial hypertension in patients transitioned from parenteral or inhaled prostacyclins: case series and treatment protocol. Pulm Circ 2016; 6: 132-135.

13 Lichtblau M, Harzheim D, Ehlken N, et al. Safety and long-term efficacy of transition from sildenafil to tadalafil due to side effects in patients with pulmonary arterial hypertension. Lung 2015; 193: 105-112.

14 Safdar Z, Thakur A, Frost A. Tolerability of switch to macitentan from bosentan in pulmonary arterial hypertension. South Med J 2017; 110: 223-228.

15 Politi M, Caruso N, Lescano J, et al. Switch from bosentan to macitentan in adult outpatients with pulmonary arterial hypertension: a real-world study. J Heart Lung Transplant 2017; 36: 4 Suppl., S166.

16 Galie N, Muller K, Scalise AV, et al. PATENT PLUS: a blinded, randomised and extension study of riociguat plus sildenafil in pulmonary arterial hypertension. Eur Respir J 2015; 45: 1314-1322. 